DZPh, Akademie Verlag, 57 (2009) 1, 37-48

\title{
Willensschwäche als Erfahrung
}

\author{
Von ANNA KUSSER (Konstanz)
}

Manchmal, so scheint mir, passiert beim Handeln Folgendes: Die Handelnde hat sich das abschließende Urteil gebildet, dass eine bestimmte Handlung die beste wäre. Sie steht zu diesem Urteil, hat aber im Moment des Handelns nicht die Motivation, die dem Urteil entsprechende Handlung auszuführen. Sie vollzieht freiwillig eine andere Handlung, die sie alles in allem für schlechter hält. Die Person ist, wie manche Philosophen sagen, willensschwach.

Ich möchte dies die einfache Beschreibung des Phänomens nennen. Sie stellt Willensschwäche als Problem der Umsetzung eines überlegten Urteils in die Tat dar. Abgesehen davon beinhaltet die einfache Beschreibung die drei klassischen Eckdaten der Willensschwäche: Eine Handlung wird für die beste gehalten (1), aber eine andere, schlechtere Handlung wird vollzogen (2), und dies freiwillig (3).

Eine Reihe von Philosophen behauptet, dass es Willensschwäche nicht gebe, genauer gesagt: gemäß der einfachen Beschreibung nicht gebe. Sie leugnen nicht, dass es Phänomene gibt, die in die Eckdaten passen, sie meinen aber, dass die einfache Beschreibung essenziell unvollständig sei und dass man, untersucht man die Phänomene genauer, die Beschreibung verändern und ergänzen müsse. Ergänzen nämlich dahingehend, dass die Person sich spätestens im Moment des Handelns von dem ursprünglichen Urteil innerlich verabschiedet habe und eine andere Einstellung wirksam geworden sein müsse. Das Wesentliche, aber auch das Problematische an dieser Position ist, dass sie es für notwendig hält, der ursprünglichen Beschreibung etwas hinzuzufügen. Diese Hinzufügung möchte ich Supplementierung nennen.

Die Unumgänglichkeit einer solchen Supplementierung leuchtet mir nicht ein. Ich möchte im Folgenden untersuchen, welche Gründe gegen die einfache Beschreibung und für die Supplementierung sprechen und inwieweit diese Gründe beinhalten, dass die einfache Beschreibung stets weichen muss.

Platon hat im Protagoras als Erster die Supplementierung vertreten. Sie ist dort Teil eines argumentativen Manövers, das darauf zielt, die in der simplen Beschreibung enthaltene Diagnose, Willensschwäche sei ein Problem der Implementierung, als Schein hinzustellen und durch die andersartige Diagnose, sie sei ein Handeln aus Unwissenheit, zu ersetzen.

Meine Kritik an der von Platon inaugurierten Traditionslinie ist von der Idee inspiriert, die Supplementierung mit der Erfahrung von Willensschwäche zu konfrontieren. Ich fasse die Supplementierung als ein Angebot zur Beschreibung dieser Erfahrung auf. Dabei möchte ich insbesondere betrachten, wie sich zwei Spielarten der Supplementierung aus der Perspektive der willensschwachen Person ausnehmen; ob sie vor dem Hintergrund ihrer Erfahrung akzeptabel und hilfreich sind. 
Ich lasse dazu eine erfundene Figur auftreten, die ich Mou Moll nenne, und die die Schwachheit in Person ist. ${ }^{1}$ Sie soll die Aufgabe übernehmen zu artikulieren, wie sich die philosophischen Angebote aus der Perspektive einer Willensschwachen ausnehmen.

\section{Die supplementierte Wertung findet immer statt - Willensschwäche als Wertmodifikation}

Ein erstes Argument dafür, die einfache Beschreibung von Willensschwäche stets zu ergänzen, könnte darin bestehen, dass ein verändertes Werturteil unmittelbar vor dem Handeln tatsächlich immer stattfindet. Sei doch ehrlich, Mou Moll! War es nicht so, dass du, als du heute morgen den Wecker zum Schweigen brachtest und, anstatt aufzustehen, dich deinem Freunde zuwandtest, war es nicht so, dass du dachtest: Nur noch ein schönes kleines Weilchen ... was kann es schaden, ich werde nachher umso rascher sein und den Zug schon noch erreichen?

Martin Seel spricht treffend von prozessualer Willensschwäche. ${ }^{2}$ Er unterscheidet erhellend zwischen

(a) der Phase ex ante: eine Handlung b wird für die beste gehalten;

(b) der Phase in situ: das Urteil wird revidiert, die Handlung s wird nun für die beste gehalten und wird vollzogen;

(c) der Phase ex post: das ursprüngliche Urteil feiert Urständ. Es wäre doch besser gewesen, b zu tun; die Handlung wird bereut.

$\mathrm{Zu}$ Recht macht die prozessuale Auffassung auf die zeitliche Erstreckung der Willensschwächephänomene aufmerksam, denn das Urteil muss gegenüber der Handlung früher sein, wenn es sie kausal bestimmen können soll. Der zeitliche Abstand zwischen Urteil und Handlung lässt Raum für „Dazwischenkommendes“: ein situativ revidiertes Urteil des Inhalts, dass s die beste Handlung ist; dieses Urteil ist dann Grund und Ursache des Vollzugs der Handlung s.

Unbestreitbar unterliegen rationale Vorsätze häufig situativen Veränderungen. Auch sind bei solchen Willensmodifikationen die Eckdaten der Willensschwäche erfüllt. Doch nicht jedes Willensschwächephänomen folgt dem prozessualen Muster. ${ }^{3}$ Ich werde argumentieren, dass diese Beschreibung nur zutrifft, wenn erstens ein modifiziertes Urteil greifbar ist und zweitens die Handlung ex post bedauert wird - zwei Bedingungen, die nicht per se erfüllt sind.

1. Das modifizierte Urteil. Das modifizierte Urteil ersetzt das ursprüngliche Urteil; es sollte daher in gleicher Weise greifbar sein wie der ursprüngliche Vorsatz. Dieser ist ein bewusstes, eine praktische Überlegung abschließendes Werturteil. Ebenso sollte auch das veränderte Urteil bewusst und das Ergebnis einer rudimentär fassbaren revidierenden Überlegung sein. Es ist daher durchaus angemessen, an dieser Stelle, wie oben geschehen, an die Introspektion der Willensschwachen zu appellieren, da ihr eine entscheidende Rolle zukommt. Hingegen kann das revidierte Urteil als mentales Ereignis nicht einfach interpoliert werden; es muss

Sie heißt so nach dem französischen mou, molle für schwach.

2 M. Seel, Ein Lob der Willensschwäche, in: ders., Sich bestimmen lassen. Studien zur theoretischen und praktischen Philosophie, Frankfurt/M. 2002, 227-244.

3 Das hebt auch Martin Seel hervor; vgl. ebd. 
durch innere Erfahrung bezeugt sein. Fehlt diese Erfahrung, so ist davon auszugehen, dass das ursprüngliche Urteil weiter unterhalten wird. Es wäre unzulässig, Mou Moll an dieser Stelle innere Erfahrungen einreden zu wollen.

Dem könnte man entgegenhalten: Es könnte doch gelten, dass man ein verändertes Urteil ins Bewusstsein ruft, wenn man die Willensschwache unmittelbar vor oder beim Handeln darauf anspräche. Würde das nicht ausreichen, um ein verändertes Werturteil anzunehmen? Dem stimme ich zu, doch ist ein solches ,schlafendes" Urteil keineswegs mit Notwendigkeit gegeben.

Nichts schließt a priori aus, dass die Willensschwache, direkt angesprochen, schaudernd festhält, dass sie gerade dabei ist, das Schlechtere zu tun. ${ }^{4}$ Sie hätte damit bezeugt, dass sie, bisher im dispositionalen Modus, nun aber greifbar, das ursprüngliche Urteil weiter unterhält.

2. Probleme mit dem Problem der Willensschwäche. Die prozessuale Auffassung fügt in die klassischen Eckdaten der Willensschwäche nicht bloß vor, sondern auch nach der Handlung ein zusätzliches Werturteil ein. Ist dieser weitere Zusatz akzeptabel? Warum dieser erneute Zusatz, könnte man nicht auf ihn verzichten?

Die Beschreibung von Willensschwäche als Modifikation von Werturteilen, insbesondere des konklusiven Werturteils, ist signifikant unterbestimmt, da sicher nicht jede Modifikation ein Fall von Willensschwäche ist. Schließlich ist es häufig gut, wenn wohlüberlegte Vorsätze einer veränderten Situation angepasst werden. Man muss also die unproblematischen Modifikationen von den problematischen abgrenzen - eine Aufgabe, die auf ein weites und kontrovers diskutiertes Feld praktischer Rationalität führt.

Vor allem muss man das spezifische Phänomen der Willensschwäche im Auge behalten. Gesucht ist eine Abgrenzung, über die Mou Moll selbst verfügt, die sie teilt. Warum? Weil sie mit ihrer Willensschwäche, nicht aber mit Wertungsmodifikationen generell, ein Problem hat. Sie verfügt also über ein Kriterium, auf Grund dessen sie zwischen unproblematischen und willensschwachen Modifikationen unterscheidet.

Nach der prozessualen Auffassung ist das Charakteristikum der Willensschwäche das nachfolgende Bedauern. Dieses ist an das ex post wiederkehrende Ex-ante-Werturteil gekoppelt und markiert den Unterschied zu unschuldigen Revisionen rationaler Vorsätze.

Was ist von dieser Sicht der Dinge zu halten? Sie wirft Fragen auf, deren Beantwortung Zusatzannahmen verlangt, die auf Seiten des Phänomens mit weiteren Ergänzungen zu Buche schlagen.

(a) Das zeitliche Problem. Es ist klar, dass die revidierte Wertung zeitlich unmittelbar vor der Handlung liegen muss. Aber wie steht es mit der erneuten Revision und dem nachfolgenden Bedauern? Wie lange darf es auf sich warten lassen? Diese Frage ist nicht unwichtig. Denn solange das Bedauern nicht eingetreten ist, hat Mou Moll kein Problem mit ihrem Handeln. Bis zum Bedauern geht sie von einer unproblematischen Revision aus. Sie selbst hatte ja befunden, dass es in der gegebenen Situation doch besser sei, nicht aufzustehen. Was, wenn sie erst nach Stunden aus dem Venusberg tritt oder gar - wie Tannhäuser - nach Jahren? Dann, möchte man sagen und wird vermutlich auch Mou Moll sagen, liegt keine Willensschwäche vor. Doch warum nicht? Die drei Phasen der prozessualen Willensschwäche sind gegeben.

(b) Kein Bedauern. Es könnte sein, dass die Ex-ante-Wertung relativ rasch wiederkehrt, doch Mou Moll nicht bedauert. Mou Moll sagt sich: Ich erinnere mich sehr gut, dass ich es heute Morgen für das Beste hielt, meinen Geliebten zu küssen. Das sehe ich zwar jetzt wieder anders: Ich wäre besser aufgestanden und pünktlich in den Zug gestiegen. Aber so ist das nun mal: Meine Einschätzungen ändern sich, je ne regrette rien. Schließlich geht nichts über das

4 Die Meinungen über die Häufigkeit dieses Phänomens gehen meiner Erfahrung nach weit auseinander. 
Prinzip, jeweils das zu tun, was man für das Beste hält. Was denn sonst, etwa das, was man für schlechter hält?

Mou Moll ähnelt jetzt der von Juliane Rebentisch in die Diskussion eingeführten platonischen Figur des wankelmütigen Demokraten. ${ }^{5}$ Die Schwankungen seiner Werturteile entgehen ihm nicht, er steht selbstbewusst zu seinen häufigen Umorientierungen, gleichzeitig lebt er nach vorne und macht sich klar, dass, auch wenn er jetzt schon wieder anders urteilt als vor kurzem noch, er doch stets das tut, was er in situ für das Beste hält - kein Grund also zu bedauern.

Der wankelmütige Demokrat ist eine interessante Figur, er ist immun gegen Willensschwäche (zumindest gegen prozessuale). Das liegt daran, dass er über kein Konzept einer rationalen Veränderung von Wertungen verfügt. Er verfügt nur über das Konzept, dass es gilt, den eigenen Wertungen gemäß zu handeln. Diesem Maßstab ist er immer treu, deshalb gibt es nichts zu bedauem. Das lässt folgenden Schluss zu: Wenn bei einer Wertmodifikation nachfolgend Bedauern eintritt, so stammt es aus anderer Quelle; es kann nicht schon daraus resultieren, dass sich die subjektiven Werte verändert haben - und sei es auch, dass die Ex-ante-Werte wieder aufleben. Denn solange den Werten gemäß gehandelt wurde, gibt es keinen Grund zu bedauern. Folgt man der Schiene, Willensschwäche als Wertmodifikation zu beschreiben, die ohne Bedauern nicht auskommt, so muss man die Quelle des Bedauerns klären.

Intuitiv scheint es klar zu sein: Mou Moll bereut, weil sie schlecht fährt; die Verwirklichung der modifizierten Werte führt - verglichen mit den Werten ex ante - zu einer Einbuße an Wohlbefinden. Mou handelt sich Scherereien ein und torpediert durch ihre Schwankung die Verwirklichung der Ex-ante-Werte, durch die sie sich besser stellen würde. Was aber, wenn sie von ihrer Willensschwäche profitiert und die schlechtere Handlung s zu einem befriedigenderen Ende führt, als es die bessere Handlung b erwarten ließ? Bei Mou Moll tritt dann ex post kein Bedauern ein. Die Folge ist, dass die prozessuale Auffassung nicht auf Willensschwäche erkennen kann. Aber wischt der glückliche Ausgang das interne Problem der Willensschwäche wirklich weg? Kann Mou Moll ohne weiteres zur Tagesordnung übergehen? Hier liegt eine weitere Komplikation für die prozessuale Auffassung, deren Behebung weitere Provisos erfordert.

Mit dem Hinweis auf Nachfolgeprobleme will ich weder in Frage stellen, dass es wichtig ist, sie zu lösen, noch behaupten, das sei nicht möglich. Klarerweise kommen situative Wertungsmodifikationen, die ex post zu Bedauern führen, immer wieder vor. Mou Moll, die Schwachheit in Person, leidet ganz gewiss auch an dieser Schwäche.

Aber ist es plausibel, all ihre Schwachheiten, insbesondere die, die sie selbst prima facie als Implementierungsprobleme ansieht, auf dieses Konto zu verbuchen? Sie selbst wird jedenfalls eine Schwachheit nicht so beschreiben, wenn sie findet, dass sich ihr Werturteil nicht verändert hatte.

Wie stellen sich die Dinge dar, wenn man auf der Schiene der ,einfachen“ Beschreibung weiterfährt? Man wird nachvollziehen können, dass Mou Moll es schlecht findet, nicht das getan zu haben, was sie für das Beste hielt und hält. Sicher, man würde sofort fragen: Wie konntest du bloß?! Wie und in welchem Sinne konnte sie handeln, ohne mit einer konklusiven Wertung dabei zu sein? Außerdem muss man darlegen, wie sich das Implementierungsproblem intern darstellt. Warum kann sie sich nicht, wie der platonische Demokrat, sagen: Macht nichts! - Wieso stellt ihr diese Erfahrung eine Aufgabe, welche Lektion hält diese Erfahrung für sie bereit?

5 Vgl. den Beitrag von Juliane Rebentisch in diesem Heft der Deutschen Zeitschrift für Philosophie: Der Demokrat und seine Schwächen. Eine Lektüre von Platons Politeia, 13-34. 
Das sind die Aufgaben; aber es gibt auch klare Chancen der Implementierungsauffassung. Mou Molls Problem liegt bereits dann auf dem Tisch, wenn ihr klar ist, dass ihr konklusives Werturteil und ihr Handeln nicht kongruieren - sie braucht keine dritte Phase, deren angemessene Länge und deren Eintreten fraglich ist. Und es braucht auch keine weiteren Hinzufügungen in Form interner Kriterien der Abgrenzung zwischen rationaler Modifikation und willensschwächeartigen Modifikationen. Nicht zuletzt stellt es einen Vorzug dar, zwischen zwei Arten praktischer Probleme unterscheiden zu können: solchen, die mit der Modifikation von Bewertungen zusammenhängen, und solchen, die mangelnde Implementierung betreffen.

Als Fazit möchte ich zwei Punkte festhalten: Auf Willensschwäche im prozessualen Sinn ist nur dann zu erkennen, wenn die veränderte Wertung greifbar ist; diese muss ein aktuelles oder abrufbares Werturteil sein, ein Werturteil, das nicht einfach interpoliert werden kann.

Dort wo das Werturteil deutlich modifiziert ist, liegt nicht unbedingt die Erfahrung von Willensschwäche vor. Der Versuch, das für Willensschwäche spezifische interne Problem im Rahmenwerk der prozessualen Auffassung einzufangen, wirft Folgefragen auf und verweist auf anders gelagerte Probleme.

\section{Die Supplementierung ist normativ gefordert - Willensschwäche als Illusion}

Der Vertreter der Supplementierung ist unbeeindruckt von dem Einwand, dass ein verändertes Werturteil nicht immer im Bewusstsein greifbar ist. Selbst wenn die Handelnde, so argumentiert er nun, an ihren Ex-ante-Wertungen festhielte, hätte sie doch andere Werturteile unterhalten sollen. Denn ihr Handeln zeige, dass sie in Wirklichkeit andere Wertungen habe. Vor dem Hintergrund dieser Wertungen war ihr tatsächliches Handeln das Beste. Es lässt sich auf diese Weise der klarsichtigen Willensschwäche Rechnung tragen, bei der die Handelnde klar sieht, dass sie das Schlechtere tut.

Folgendes Szenarium ergibt sich: Ex ante hält Mou Moll es für besser, b zu tun, und bleibt auch vorderhand dabei; in situ vollzieht sie die schlechtere Handlung s; ex post redet sie von Willensschwäche, um nicht zu sagen: Sie redet sie sich darauf heraus.

Mindestens ex post, so der Vorschlag, sollte sie ihre faktische psychische Verfassung zur Kenntnis nehmen. Wenn ihr Bewusstsein auf Grund eigentümlicher Mechanismen - Irrtum oder Selbsttäuschung - kein getreuer Spiegel ihrer tatsächlichen Werthaltungen ist, umso schlechter. ${ }^{6}$

Es scheint, dass hier eine zusätzliche Evaluation ,nicht wirklich“" supplementiert, sondern nur eine bereits vorhandene psychische Werthaltung benannt wird, welche Benennung allerdings eingefordert wird. Denn - so diese Auffassung - die Erfahrung von Willensschwäche macht Mou Moll mit ihren wirklichen Wertungen bekannt und sollte zur Korrektur falscher, illusionärer Selbsteinschätzungen führen. Dem illusionären Selbstbild wird in der Phase der praktischen Überlegung gefrönt - einem Selbstbild, das sich als haltlos erweist und das in Wirklichkeit von anderen, nämlich den handlungswirksamen Werthaltungen unterfüttert ist.

Bringen wir Mou Moll ins Bild, welchen Vorschlag beinhaltet dies für sie? Sie versucht, sich herauszureden, sagt der supplementierende Philosoph, doch das lässt er ihr nicht durch-

6 Nach Ursula Wolf vermischen sich häufig Selbsttäuschung und Wertmodifikation; ich betrachte hier diese beiden Diagnosen getrennt, um die jeweils implizierten Annahme herausarbeiten zu können; vgl. U. Wolf, Zum Problem der Willensschwäche, in: Zeitschrift für philosophische Forschung, 39 (1985), 21-33. 
gehen. ${ }^{7}$ Er möchte, dass sie etwas in dieser Art sagt: Die Wertungen, auf deren Grundlage ich dachte, dass $b$ die beste Handlung sei, sind nicht meine wirklichen Werthaltungen. Hätten meine Werturteile meine wirklichen Werthaltungen zum Ausdruck gebracht, dann wäre mir bereits ex ante klar gewesen, dass nicht $\mathrm{b}$, sondern $\mathrm{s}$ die beste Handlung war, ich $\mathrm{s}$ irgendwie für besser hielt, auch wenn ich klarerweise so nicht gedacht habe.

Ist diese Sicht der Dinge annehmbar, insbesondere für Mou Moll?

Man könnte als erstes fragen, wie es sich erklärt, dass Mou Moll Wertungen unterhält, ohne davon zu wissen. Verbirgt sie ihre Werthaltung vor sich? Ich möchte diesen Punkt auf sich beruhen lassen. Ich werde mich nur an die Behauptung halten, dass Prozesse am Werk sind, aus denen Werturteile resultieren, die Wertungen falsch wiedergeben. Willensschwäche stellt sich hier als Illusion dar. Ich werde argumentieren, dass diese Sicht der Dinge inkonsistente Annahmen und Konsequenzen beinhaltet.

1. Rationalisierung. Eigene Handlungen figurieren hier als Gründe, sich diejenigen Werthaltungen zuzuschreiben, die sich im Handeln offenbaren, da dieses auf die Realisierung dieser Werte hingeordnet ist. Ein solcher Rückschluss ist voraussetzungsvoll, aber in den Sozialwissenschaften, der Psychologie, im Kriminalroman und nicht zuletzt im Alltag an der Tagesordnung. Man unterstellt dabei Handlungen als rational; man identifiziert eine Beschreibung, unter der eine Handlung als intentional gelten kann, und erschließt - vor dem Hintergrund von Annahmen über vorhandenes Wissen oder Meinungen -, worauf die Handlung abzielte. Die wirklich angestrebten Zwecke werden dem Subjekt als Werthaltungen zugeschrieben. Das sei einmal für den Moment akzeptiert.

So auch bei Mou Moll: Wir weisen ihr lückenlos nach, wie wichtig ihr jene Viertelstunde doch war - in Wirklichkeit wichtiger als ihre terminliche Verpflichtung; wusste sie doch ganz genau, dass der Zug verpasst, der Termin platzen würde und so weiter. Was sagt Mou Moll dazu? - Mir war und ist mein beruflicher Erfolg enorm wichtig; bedenk doch nur, was ich alles getan habe, um den Termin zu Stande zu bringen. Ich kann mir selbst nicht erklären, warum ich nicht aufgestanden bin. Wie auch immer: Dass ich nicht aufgestanden bin, ist für mich kein Grund, von meiner Auffassung, dass ich besser aufgestanden wäre, abzugehen und stattdessen nun zu meinen, das sei das einzig Richtige gewesen, weil mir - wie man mir weismachen will - anderes wichtiger gewesen sein soll. Sicher, wenn ich die Dinge so sehen würde, dann wäre mein Handeln für andere, aber auch mich selbst, verständlicher und rationaler - doch das wäre eine klassische Ex-post-Rationalisierung. Die Tendenz, die Dinge so zu sehen, ist mir nicht unbekannt, doch hüte ich mich, dem zu folgen. Umso erstaunter bin ich, dass man mir diese Sicht empfiehlt. ${ }^{8}$

Mou Molls Weigerung führt plastisch vor Augen, dass eigenes vergangenes (aber auch vorhergesehenes zukünftiges) Handeln keinen guten Grund bildet, passende Werturteile zu unterhalten.

Nun könnte man einwenden: Es geht nicht darum, auf Werturteile, sondern auf wirkliche Werthaltungen zu schließen. Wer meint, dieser Schluss sei nicht möglich, geht von dem Dogma

$7 \quad$ Vgl. J. Schälike, Willensschwäche und Selbsttäuschung. Über die Rationalität des Irrationalen und das Verhältnis von Evaluation und Motivation, in: Deutsche Zeitschrift für Philosophie, 52 (2004), 361-379, bes. $376-378$.

8 Vgl. A. McIntyre, What is wrong with weakness of will?, in: Journal of Philosophy, 103 (2006), 284-311, bes. 285-288. McIntyre macht einen interessanten Vorschlag zur gemeinsamen Wurzel von Willensschwäche und Rationalisierung, sieht aber ebenfalls Rationalisierung als einen zur Willensschwäche gegebenenfalls noch hinzukommenden Fehler. 
aus, Werthaltungen müssten immer bewusst und in Urteile gegossen sein. Könnte das nicht falsch sein? Vielleicht. Gleichwohl möchte man hier über den Zwischenschritt der Supplementierung anderer als der bewussten Werthaltungen dort ankommen, dass die bewussten Werturteile revidiert werden müssen. Das läuft am Ende darauf hinaus, dass, weil Handlungen Evidenzen für Werthaltungen sind, der Verweis auf Handlungen Gründe bietet, Werturteile passend zu verändern. Dem liegt die Annahme zu Grunde, dass Werturteile Überzeugungen über eigene Werthaltungen sind und deshalb diese wiederzugeben haben. Diese Annahme muss aufgegeben werden, denn sie führt zu der inakzeptablen Forderung, Werturteile den erschlossenen Werthaltungen anzupassen.

2. Handlungsdispositionen und Wertungen. Zu Recht rückt Mou Moll nicht von ihren Werturteilen ab. Doch ein Problem bleibt: Sie weiß, dass die anderen ihre Werturteile an ihrem Handeln messen und darauf achten, wie viel sie ,wirklich“ wert sind. Außerdem: Mou Moll tut so, als verstünde sie nicht, was da passiert ist. Aber Mou, so schwer ist das nicht! Andere verstehen sehr gut: Honigmund war wichtiger.

Wenn für dritte Personen der Rückschluss von den Handlungen auf die Präferenzen möglich ist und zu gültigen Ergebnissen führt, warum kann Mou sich ihm entziehen? Gibt es nicht doch unbewusste Werthaltungen? Warum sollte etwas, was aus der Perspektive anderer zutrifft, aus ihrer Perspektive falsch sein?

Die Lösung ist diese: Auch für Mou Moll selbst ist der Rückschluss gültig. Von anderen - aber auch ihr selbst - wird auf diese Weise jedoch auf Handlungsdispositionen, nicht auf Wertungen geschlossen. Mou Moll hat ebenso wie andere Personen Überzeugungen über ihre Handlungsdispositionen. In der Regel glaubt sie - glauben auch andere -, dass sie disponiert ist, so zu handeln, dass ihre Werte verwirklicht werden. Sie wird jeweils das tun, was gemessen an ihren Werten das Beste ist; denn, so glaubt sie, ihre Werte motivieren ihr Handeln. In der Regel stimmt das. Daher kann im Normalfall von Dritten indirekt über die Handlungen auf die Wertungen geschlossen werden. Mou Moll nimmt aber bei der Bildung und Revision ihrer Werturteile nicht diesen Weg.

Macht sie die Erfahrung der Willensschwäche, so merkt sie, dass ihre Werturteile sie nicht entsprechend motivieren; sie ist nicht entsprechend disponiert zu handeln. Das zwingt sie nicht, ihre Wertungen zu revidieren; doch sie muss die ihnen anhängende Überzeugung revidieren, dass sie motiviert ist, ihre Werte zu verwirklichen. Ganz ebenso wie Dritte erschließt Mou aus ihrem Handeln, welche Ziele sie in ihrem Handeln ansteuert, oder genauer: dass die Hierarchie der von ihr de facto angesteuerten Ziele von ihren Evaluationen abweicht. Diese Überzeugungen über ihre Handlungsdisposition oder ihre Motivation können aber nicht ohne weiteres ihre Werturteile verändern oder ersetzen.

3. Wiederum: Probleme mit dem Problem der Willensschwäche. Wenn im Zentrum von Willensschwäche eine Illusion stünde, so wäre sie ein Fall theoretischer, nicht praktischer Rationalität. Entsprechend dieser Problemdiagnose bestünde die Therapie darin, von illusionären falschen Überzeugungen zu richtigen, die Tatsachen wiedergebenden, überzugehen. Doch ist es plausibel, dass das Problem der Willensschwachen dann behoben ist, wenn Mou Moll anders über sich denkt? ${ }^{9}$

9 Hier besteht ein signifikanter Unterschied zur platonischen Supplementierung. Nach Platon handelt der Willensschwache aus Unwissen und ist aufgerufen, das einzusehen. Er handelt aber nicht aus Unwissen über sich selbst, sondern aus Unwissen über die wahre Größe der Lust. 
Noch merkwürdiger ist, dass in der Konsequenz dieses Ansatzes für sie sowieso kein praktischer Anlass besteht, das Problem anzugehen. Denn ihren wirklichen Werthaltungen hat sie in ihrem Handeln immer schon nachgelebt. Genau deshalb, weil ihre Werte nach dieser Sicht jederzeit ihr tatsächliches Handeln bestimmen, kann man sie daran ablesen. Ihre Werturteile und die konklusive Handlungsbeurteilung spielen demnach keine praktische Rolle; es sind bloße Oberflächenphänomene, die ihr Handeln nicht wirklich bestimmen. Diese Implikation scheint mir ein sehr gravierender Makel dieser Auffassung zu sein.

Könnte es nicht sein, dass nur bei Willensschwäche die wirklichen Wertungen das Handeln bestimmen, während im Normalfall, wenn Mou Moll tut, was sie für das Beste hält, die Werturteile das Handeln bestimmen? Das wäre seltsam: Ihr Problem läge dann darin, dass leider manchmal ihre tatsächlichen Wertungen ihr Handeln bestimmen.

Fazit: Die Beschreibung von Willensschwäche als Illusion ist eine Konzeption voller innerer Ungereimtheiten. Insbesondere kann die Forderung, willensschwaches Handeln zu rationalisieren - eine Forderung, die in die Konzeption eingebaut ist -, vor der Erfahrung der Willensschwäche nicht bestehen. Wir sollten Mou Molls Reaktion darauf auf theoretischer Ebene nachvollziehen und diese Form der Supplementierung definitiv verweigern. Das bedeutet, dass man Fälle von klarsichtiger Willensschwäche nicht in dieser Weise auffassen kann.

Zwei wichtige Einsichten hat die Auseinandersetzung mit dieser Position aber erbracht:

Werturteile sind keine Überzeugungen über eigene Werthaltungen und müssen nicht nach den faktisch verfolgten Zielen gemodelt werden. Und: Es ist richtig, dass sich aus dem Handeln von Personen die von ihnen verfolgten Ziele erschließen lassen.

\section{Freiwilligkeit}

Ein bestimmendes Motiv hinter den beiden Formen der Supplementierung ist die Rettung des Dogmas, dass Handlungen nur freiwillig sein können, wenn sie von der Person vorweg als alles in allem beste angesehen wurden. ${ }^{10}$

Klarsichtige Willensschwäche zwingt scheinbar endgültig dazu, dieses Dogma aufzugeben. Hier nun setzt ein weiterer Rettungsversuch ein: Man versucht zu zeigen, dass klarsichtige Willensschwäche immer dazu zwinge, die Freiwilligkeit der Handlung preiszugeben. Ist die Konstellation gegeben, dass ein Handelnder klarsichtig b für die beste Option hält, aber s tut, so folgt, dass er s nicht freiwillig tut.

Mou Moll nimmt dieses Angebot zunächst nicht ungern entgegen; sie wird aus der Verantwortung entlassen - das erleichtert.

Keine Frage, es gibt Fälle, in denen vieles für pathologischen inneren Zwang spricht und die Handlung deutlich unfreiwillig war. Dann handelt es sich - das ist unkontrovers - nicht um Willensschwäche. Zur Diskussion stehen die uneindeutigen Fälle, das heißt Fälle, die dem ersten Zugang nach freiwillig aussehen. Hier nun soll, bei genauerem Hinsehen, die Freiwilligkeit der Handlung Schein sein.

Mou Moll ist ein uneindeutiger Fall. Ihr sind Zweifel gekommen. Mit einem Schlag soll ihr ganzes Problem verschwunden sein, das ist unglaubhaft. Sie ist mit ihrem Handeln gar nicht zufrieden und möchte dem vorbauen, dass ihr wieder so etwas passiert. Wo ist der Fehler passiert? War die Handlung s nicht die schlechtere? Doch - das schlechte Ergebnis bestätigt

10 Vgl. hierzu auch den Beitrag von Matthias Vogel in diesem Heft der Deutschen Zeitschrift für Philosophie: Fehler im Haus der Vernunft, 71-93. 
sie. Hier kann der Fehler nicht liegen. Stand es ihr nicht offen, s zu unterlassen? Das sagt man ihr. Dann hätte sie s gar nicht erst in Betracht ziehen dürfen. Genau daran hat sie aber Zweifel. Denn es steht fest, dass die Handlung in ihrem Verlauf unter ihrer Kontrolle stand; sie hätte, meint sie, es durchaus lassen können, mit dem Honigsaugen anzufangen. Dass sie es tat und gleichzeitig meinte, es sei schlechter, heißt nicht, dass sie die Handlung nicht kontrollieren konnte. Sie sieht daher keinen Grund, Honigsaugen aus dem Bereich der ihr in solchen Situationen offen stehenden Optionen zu entfernen.

Solange die Kontrollannahme gilt, ist das Problem aus Mou Molls Sicht nicht gelöst. Die Gegenseite müsste nun zeigen, dass jeder Handelnde sich irrt, wenn er meint, er habe Kontrolle über eine Handlung, die er vollzieht, obwohl er sie für schlechter hält. Weiterhin muss gezeigt werden, dass der dabei verwendete Kontrollbegriff genau der ist, den der Handelnde braucht, um eine Handlung in den Bereich der offenen Optionen aufzunehmen. "

Ich komme zu dem Schluss, dass die Behauptung, die Freiwilligkeitsbedingung müsse bei klarsichtiger Willensschwäche immer zurückgezogen werden, keineswegs evident ist. Insbesondere muss diese Rücknahme auf Erwägungen beruhen, die mit der Innensicht eines Handelnden, der die Erfahrung der Willensschwäche macht, kompatibel sind.

\section{Evaluation und Motivation}

Klarsichtige Willensschwäche kann, so habe ich argumentiert, nicht durch Supplementierung und durch Rückzug der Freiwilligkeitsbedingung in den rationalistischen Rahmen eingefügt werden. Sie ist der paradigmatische Fall, der eine Ausformulierung des in der einfachen Beschreibung enthaltenen Konzepts mangelnder Implementierung herausfordert. Auch wenn dieser paradigmatische Fall in aller Reinheit nicht häufig vorkommt, gewinnt man an ihm eine Beschreibung, die sich auch an weniger krassen Fällen bewährt.

Die zentralen Begriffe von Evaluation und Motivation sind bis zu einem gewissen Grade Termini, die von ihrer ungeregelten Verwendung im Alltag abweichen. Sie lassen sich aber unschwer kontextuell an alltägliche Wendungen anschließen. ${ }^{12}$

Unter der Evaluation oder auch den Evaluationen einer Person verstehe ich ihre bewussten subjektiven Werte, die sie in Werturteilen zum Ausdruck bringt; es geht um Urteile wie: ... ist mir wichtig, ist mir unwichtig, möchte ich erreichen, ziehe ich vor, strebe ich an und so weiter. Die Wertungen beziehen sich auf Propositionen: Es ist immer etwas, ein Sachverhalt, der wichtig ist. Die subjektiven Werte sind in der Regel bewusst oder können bewusst gemacht werden. Sie sind die Ausgangspunkte der praktischen Überlegung, die darauf zielt, die Handlung zu finden, die diese Werte am besten, das heißt am umfangreichsten und sichersten, verwirklicht. Die Konklusion einer praktischen Überlegung ist die abschließende Bewertung einer Handlung als in dieser Hinsicht bester. Damit ist das Ende der Überlegung markiert, und es wird zum Handeln - zur Implementierung - geschritten.

Evaluationen sind immer graduiert - es handelt sich um Präferenzen. Das bedeutet nicht, dass es einer Person immer schon und jederzeit klar wäre, dass und wie stark sie einen Sach-

11 Es ist klar, dass hier intrikate Probleme lauern. Eine sorgfältige Analyse der begrifflichen Landschaft - wenn auch im Hinblick auf konträren Positionsbezug - bietet: J. Schälike, Selbstkontrolle, Synchrone contra diachrone Analyse von motivationalem Zwang und Willensschwäche, erscheint demnächst in: Studia Philosophica, 2009.

12 Etwas ausführlichere Darlegungen und Verteidigungen gegen rivalisierende Ansätze finden sich in:

A. Kusser, Dimensionen der Kritik von Wünschen, Frankfurt/M. 1989, 106-163. 
verhalt anderen Sachverhalten vorzieht. Nur: Wenn sie eine praktische Überlegung bis zum Ende führen will, dann muss sie von einer definierten Vorzugstruktur ausgehen. Wann immer es ein Urteil derart gibt, dass eine bestimmte Handlung die beste sei, beruht das auf graduierten Evaluationen. Diese liegen als letzte intrinsische Evaluationen oder als bereits zweckrational überdachte (etwas ist mir wichtig, um einer anderen Sache willen) der praktischen Überlegung zu Grunde. Evaluationen sind keine Überzeugungen über eigene Werthaltungen, sondern propositionale Einstellungen eigener Art.

In der Ex-ante-Phase der Willensschwäche spielen die Evaluationen die tragende Rolle; auf ihnen beruht das Urteil, b sei die beste Handlung, - ein Urteil, das selbst eine abschlieBende Evaluation darstellt.

Evaluationen hängt normalerweise die Überzeugung an, man sei entsprechend motiviert. Solche Überzeugungen sollen konsonant zu den Bewertungen heißen.

Was beinhalten sie? Man glaubt, man werde das Nötige tun, um den Wert zu verwirklichen; „entsprechend“ heißt: Man sei in dem Grade motiviert, wie es dem Rang in der Präferenzordnung entspricht. Das läuft darauf hinaus, dass man glaubt, bereit zu sein, die handelnde Verwirklichung des Wertes zurückzustellen oder vorrangig zu betreiben, je nach seinem relativen Rang.

Wie sieht die Lage in situ aus? Über die beste Handlung besteht Klarheit, aber man tut sie nicht. Die Zeit des Handelns verstreicht, der Zug fährt ab, spätestens jetzt muss Mou Moll ihre Überzeugung revidieren: In Wirklichkeit ist sie nicht entsprechend motiviert.

Daraus resultiert ein kognitiv dissonanter Bewusstseinszustand: Etwas hoch zu bewerten und gleichzeitig von sich selbst zu glauben, man werde nicht entsprechend die Finger rühren, wenn's drauf ankommt - das passt nicht zusammen. Ein solcher Bewusstseinszustand schafft einen Veränderungsbedarf.

Der krasse klarsichtige Fall von Willensschwäche wirft ein interessantes Licht auf Fälle im Umfeld: Mou Moll merkt, dass es nicht entsprechend läuft. Sie wendet jetzt Techniken der Selbstmotivation an: Sie visualisiert ihren geschäftlichen Erfolg; es folgt eine strenge Selbstermahnung und was es da sonst noch so gibt. Gut möglich, dass diese mentalen Techniken zum Erfolg führen und sie aufsteht.

Oder aber ein anderes Programm läuft ab: Mou beginnt im Vorfeld zu rationalisieren: Ich bin zu sehr auf meinen Geschäftserfolg fixiert, so wichtig ist mir das auch wieder nicht, es gibt noch anderes im Leben und so weiter: Kurz und gut, sie versucht, ihre Bewertung zu drücken und so der vorweg als schwach eingeschätzten Motivation anzupassen. Es handelt sich hier um eine Art Ex-ante-Supplementierung - diesmal durch Mou selbst. Der Skandal mangelnder Implementierung wird im letzten Moment normalisiert. Solche Prozesse zielen darauf, die drohende Diskrepanz abzuwenden, indem an der Evaluation oder an der Motivation gedreht wird. Die Unstimmigkeit von Evaluation und Motivation wird im Vorfeld abgebogen, wodurch die Erfahrung der Willensschwäche vermieden wird.

Angenommen, die präemptive Normalisierung in situ klappte nicht. Ex post ist klar: Mou Molls Annahme, sie sei entsprechend motiviert, ist falsch, ihre Willensschwäche ist nun offenkundig. Wo liegt nun das Problem für Mou? Hat sie überhaupt notwendig ein Problem, kann sie nicht mit der Achsel zucken und sagen, na ja, Evaluation und Motivation, das sind eben zwei Paar Stiefel? Niemand kann ihr das verbieten. Nur, nächste Woche steht schon wieder ein morgendliches Meeting an. Wie will sie dann ihre Ziele weiterverfolgen? Wird sie einfach den Wecker stellen und glauben, dass sie aufstehen wird? - Sie sagt sich vielleicht: Es war ein einmaliger Ausrutscher. Damit bekräftigt sie: Ja, ich glaube, dass ich entsprechend motiviert bin. Wenn aber zu viel dagegen spricht, und sie diesen Glauben nicht aufrechterhalten kann, wird es schwierig für sie. Sie kann dann nicht mehr davon ausgehen, dass sie so handeln wird, wie sie glaubt, dass es zur Erreichung ihrer evaluativen Ziele notwendig ist. Dann wird die 
praktische Überlegung sinnlos; ihre Planung verliert in dem Maße ihren Punkt, wie im Voraus klar ist, dass sie nicht umgesetzt wird.

Das heißt: Vom Standpunkt der praktischen Überlegung muss Mou Moll von der Kongruenz ausgehen. Wenn das nicht der Fall ist, hat sie Grund, ex post Maßnahmen zu ergreifen, die für Kongruenz sorgen.

Hier gibt es verschiedene Möglichkeiten: Mou Moll kann einmal zusehen, dass die Motivation, ein Ziel zu erreichen, dessen Bewertung entspricht. Sie würde dann die Motivation der Evaluation anpassen. Sie kann aber auch anstreben, dass ihre Evaluationen der faktischen Motivation entsprechen. Auch eine Kombination der beiden Richtungen ist möglich; denn wichtig ist vor allem, dass sie zu Evaluationen kommt, die sie motivieren beziehungsweise von denen sie das glauben kann.

Die Anpassung der Evaluation an die Motivation, so mag man einwenden, ist fragwürdig. Ist es nicht zu billig, die Ziele einfach herunterzustufen, als Reaktion darauf, dass man versäumte, ihnen nachzuleben? Mou Moll geht der Anstrengung aus dem Weg, außerdem wird sie es beruflich zu nichts bringen.

Zunächst zum ersten Punkt: Tatsächlich, Mou muss sich jetzt weniger anstrengen, aber daran ist an sich nichts Schlechtes, abgesehen von Punkt 2, den ich unten aufgreife. Warum sollte sie sich anstrengen, für Ziele, die ihr nicht wichtig sind?

Auch ist die Veränderung der Evaluation manchmal nicht so einfach zu haben, wie es scheint. Wenn tiefsitzende intrinsische Ziele betroffen sind, die die Pfeiler des Selbstbildes bilden, kann es subjektiv schwierig sein, von solchen Zielen Abstand zu nehmen. Zu beachten ist außerdem, dass es sowohl um evaluative Rückstufung wenig motivierender Ziele als auch um evaluative Hochstufung überproportional motivierender Ziele geht, wenn die Evaluation der Motivation angepasst wird.

In der Tradition ist der Weg der Anpassung der Motivation an die Evaluation privilegiert worden. Das mag damit zusammenhängen, dass ein positives Vorurteil zu Gunsten der bewussten Wertungen vorherrscht, weil sie vernunftnah sind. Gleichzeitig ist es vom Ziel her verständlich. Das Ziel ist, metaphorisch gesprochen, die volle Herrschaft der Werte, denn davon muss man vom Standpunkt der praktischen Überlegung ausgehen. Doch die volle Herrschaft der Evaluation ist immer dann erreicht, wenn Evaluation und Motivation kongruent sind, ganz gleich wo dieser Punkt liegt. Man kann diesen Zielpunkt daher auch in der anderen Richtung suchen - dort wo sich die faktische Motivation aufhält.

Ich habe oben ein Argument für die Anpassung der Motivation an die Evaluation liegen gelassen. Die Verwirklichung der evaluativen Ziele, so lautet es, stellt die Handelnde besser, die Evaluationen sind daher der Maßstab. Mou Moll wird es beruflich zu nichts bringen, wenn sie ihrer Motivation nachlebt, und das wird sie am Ende frustrieren. Hier ist die Unterstellung am Werk, dass die Verwirklichung der evaluativen Ziele befriedigender ist als das Erreichen der motivationalen Ziele. Mit den Evaluationen verbindet sich der wahrhaft größere Nutzen. ${ }^{13}$

Mit diesem Argument kommt ein neuer Gesichtspunkt ins Spiel: Die bessere Handlung ist nicht bloß besser, weil sie die evaluativen Werte am umfangreichsten und sichersten verwirklicht; sie ist auch besser im Hinblick auf die Befriedigung, die Mou in der Verfolgung dieser Ziele erlangen wird. ${ }^{14}$

13 Von dieser Unterstellung geht Luc Bovens' monologisierender Akratiker aus; vgl. L. Bovens, Die beiden Gesichter der Anonymen Akratiker, in: Th. Spitzley (Hg.), Willensschwäche, Paderborn 2005, 218-224.

14 Subjektive Wertungen sind in der Regel mit der Überzeugung verbunden, dass mit Verwirklichung der Werte ein entsprechender Nutzen, im Sinne von subjektivem Wohlbefinden, erreicht wird. Diese 
Angenommen, die schlechtere Handlung wäre im Hinblick auf den Nutzen in Wirklichkeit die bessere. Wäre Willensschwäche in solchen Fällen nicht ein Segen? Gäbe es dann noch ein Problem der Willensschwäche? ${ }^{15}$

An der Erfahrung der Willensschwäche und dem damit verbundenen Problem mangelnder Umsetzung eigener evaluativer Ziele würde ein positiver Ausgang nichts ändern. Zeigt die Erfahrung am Ende, dass die schlechtere Handlung und die mit ihr verbundenen motivationalen Ziele - gemessen am erreichten Nutzen - die besseren sind, so wäre das ein Grund, die Evaluation der Motivation anzupassen. Stellt sich die Handelnde hingegen mit dem Erreichen ihrer motivationalen Ziele vergleichsweise schlechter, so ist das ein Grund, an der Evaluation festzuhalten und die Motivation anzupassen. Das Hinzuziehen der Nutzenbetrachtung liefert einen Gesichtspunkt zur Wahl der Anpassungsrichtung.

Unabhängig vom guten oder schlechten Ausgang - der ja in der Ferne liegen mag - beinhaltet die Erfahrung von Willensschwäche eine Aufforderung zur Modifikation subjektiver Werte. Denn durch diese Erfahrung realisiert die Handelnde, dass einzelne ihrer Ziele inkongruent sind. Diese Ziele stehen in der evaluativen Wertpräferenz nicht - noch nicht - an der richtigen Stelle. Die richtige Stelle beziehungsweise eine richtige Stelle - denn es ist nicht ausgeschlossen, dass es mehrere gibt - ist dann gefunden, wenn ein Ziel entsprechend seinem evaluativen Rang motiviert. So gesehen, hält Willensschwäche für Personen eine wichtige Erfahrung bereit, auf dem Weg zu ihren je eigenen ,wahren"Werten.

\title{
Dr. Anna Kusser, Universität Konstanz, Fachbereich Philosophie, 78457 Konstanz
}

\begin{abstract}
In the Platonic tradition, weakness of will is not the failure to implement a practical judgement, but a different sort of failure. In describing that different failure, philosophers typically supplement the phenomenon of akrasia by an additional judgement. It is argued that with this supplementation, the experience of weakness of will becomes distorted. Using instead a suitably designed distinction between evaluation and motivation allows for a more direct description. On the basis of this description akrasia may be understood as an experience which helps persons to find their true subjective values.
\end{abstract}

Überzeugung kann ebenso wie die konsonante Überzeugung über die eigene Motivation falsch sein. Auch das konstitutiert einen ,internen“ Problemfall für die Handelnde. Vgl. dazu A. Kusser, Dimensionen der Kritik von Wünschen, a. a. O., 185-193.

15 Der unerwartete Segen eines glücklichen Ausgangs akratischer Episoden ist wohl zu unterscheiden von dem kostenreich organisierten Extra-Profit, durch den sich L. Bovens' Akratiker sanieren will. 